\title{
OBSTACLE DETECTION IN ROAD SCENE BASED ON ROAD DETECTION AND MOTION ANALYSIS
}

\author{
Cédric Demonceaux ${ }^{*, * *, 1}$ Alex Potelle* \\ Djemâa Kachi-Akkouche* \\ * C.R.E.A., EA 3299, \\ 7, rue du moulin neuf, 80000 Amiens, France \\ ** L.A.M.F.A., UMR 6140, \\ 33, rue Saint-Leu, 80039 Amiens Cedex 1, France
}

\begin{abstract}
This paper deals with the problem of obstacle detection in traffic applications. The proposed device allows a diver to receive the current road and vehicle environment information. The environment perception is performed through a fast processing of image sequence acquired from a vision system embedded in a vehicle. The approach is based on frame motion analysis. Firstly, the road motion is computed through a fast and robust wavelets analysis. Then, we detect the areas which have a different motion thanks to a bayesian modelization. Results shown in the paper prove that the proposed method permits the detection of any obstacle on a road in various image conditions.
\end{abstract}

Keywords: Motion estimation, road detection, obstacle detection.

\section{INTRODUCTION}

The ability to detect obstacles and the degree of danger is essential for vehicle navigation. The work described in this paper is part of a project to develop a cooperative perceptual system for traffic applications. It should inform the driver of the presence of obstacles and critical situations on the road and help him to adapt in advance his behavior on the road.

The techniques used in the obstacle detection may vary according to the definition of the obstacles. When the definition is reduced to a specific object (vehicle, pedestrian), the detection can be based on search for specific patterns, possibly supported by features such as texture, shape (Lutzeler and

\footnotetext{
1 This work was supported by "Région Picardie" under the project "Pôle DIVA"(005: "Perception coopérative intervéhicules pour la détection d'imprévus dans un milieu routier")
}

Dickmanns, 1998) (Broggi et al., 2000), symmetry (Kuehnle, 1998) or the use of an approximate contour. In this case, the processing can be limited to the analysis of a single image. On the other hand, when the obstacle definition is more general, obstacle detection is confined to the area identification in which the vehicle can safely move. In this case, two approaches are generally used: analysis of the optical flow (Lefaix et al., 2002),(Kruger et al., 1995) and stereo vision (Bertozzi and Broggi, 1998). These techniques are huge calculation cost and sensitive to vehicle motion. Moreover, optical flow techniques fail when obstacles have small or null speed. In this paper, we describe a new strategy to detect obstacles in road sequence from a single camera mounted on a vehicle. Our method is based on optical flow estimation. In order to obtain a fast algorithm, we detected the interesting area in the image using a method which detects the road. Then, we estimate 


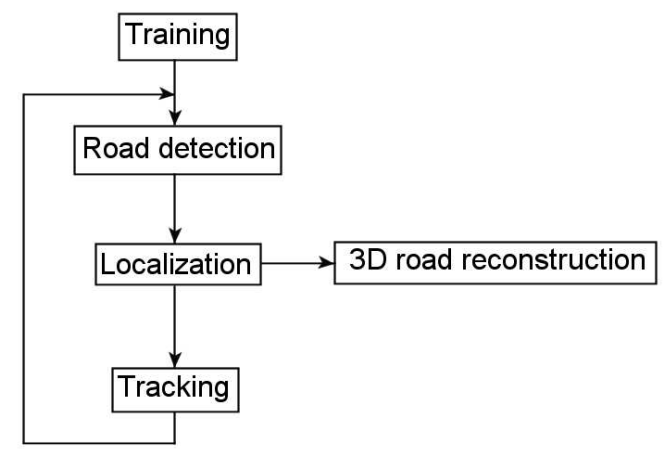

Fig. 1. Algorithm principle

the motion using wavelets analysis of the optical flow equation only on the area of interest. To detect the obstacles which have small speed, we modelize the road velocity by a quadratic model. Then, to achieve a robust algorithm, we use a fast bayesian modelization instead of a simple threshold between the expected and real velocity fields. The method proposed decomposes into three steps: road detection, road motion estimation and obstacle detection on the road. The paper is organized as follows : section 2 briefly describes the road detection in the image, section 3 presents a robust algorithm which enables to estimate the dominant motion, i.e., the road motion. In section 4 , we show how to solve the problem of obstacle detection. Some results are presented in section 5 .

\section{ROAD DETECTION IN IMAGE SEQUENCE}

At this step of the work, we consider the following problem: extracting a part of the image which corresponds to the road surface.

Our goal is to obtain a reliable three-dimensional shape of the road in order to detect dangerous obstacles. To achieve this, we use a method developed by Chapuis and al (Chapuis et al., 2002),(Chausse et al., 2000). This approach, described in Figure 1, is fast and robust. Moreover, it enables us to detect marked or not-marked road and uses an on-board single monocular monochromatic camera.

The algorithm is composed of five steps : training, road detection, localization and tracking. The $3 \mathrm{D}$ reconstruction profile of the road is computed after the localization. The approach is based on a recursive recognition of the road sides (or road lines) driven by a probabilistic model (vector X and its covariance matrix $C_{X}$ ). $\mathrm{X}$ is composed of 18 image parameters (9 image $\mathrm{x}$-coordinates for the left road side/lines and 9 for the right, denoted $u$ ) and of 5 three-dimensional parameters (lateral vehicle position $x 0$, vehicle steer angle $\psi$, camera inclination angle $\alpha$, lateral road curvature $C$ and road width $L)$.

$X=\left(X_{d}, X_{l}\right)^{t}$ where $X_{d}=\left(u_{1 l}, \ldots, u_{9 l}, u_{1 r}, \ldots, u_{9 r}\right)^{t}$ and $X_{l}=\left(x_{0}, \psi, \alpha, C, L\right)^{t}$.

$C_{X}=\left(\begin{array}{cc}C_{X_{d}} & \vdots \\ \vdots & C_{X_{l}}\end{array}\right)$ where $C_{X_{d}}\left(\right.$ resp. $\left.C_{X_{l}}\right)$ is a covariance matrix of $X_{d}$ (resp. $X_{l}$ )

\subsection{Training}

It's an off-line process. The goal is to compute initial vector model $X(0)$ and its covariance matrix $C_{X}(0)$. To do that, Chapuis and al use a simple 3D road model projected randomly a great number of times in the image (following fixed intervals). The equation of a road side/line in the image is defined by:

$u=e_{u}\left[-\frac{e_{v} Z_{0}}{2\left(v-e_{v} \alpha\right)} C+\frac{v-e_{v} \alpha}{e_{v} Z_{0}}\left(x 0+\gamma \frac{L}{2}\right)-\psi\right](1)$

where

$u, v$ are pixel coordinates in the image,

$\gamma=1$ (left road side/line),

$\gamma=-1$ (right road side/line),

$e_{u}=\frac{f}{d u}, e_{v}=\frac{f}{d v}$ with f: focal distance of the camera, $d u, d v$ : width and height of a pixel,

$Z_{0}$ is the height of the camera.

\subsection{Road detection}

The road detection procedure is iterative. After the training step, an optimal interest zone is chosen to initialize the algorithm (depth $p=0)$. If a piece of road line or road side is found, the model is updated according to equation (2) and a new optimal zone is located (now depth $p=p+1$ ). The process is finished when $p=p f(p f=9$ in our case) valid detections have been found.

$$
\left\{\begin{array}{l}
X_{d}(p)=X_{d}(p-1)+K[y(p)-x(p-1)] \\
C_{X_{d}}(p)=C_{X_{d}}(p-1)-K H C_{X_{d}}(p-1)
\end{array}\right.
$$

where

$y(p)$ are measurement at depth $p$,

$x(p-1)$ are measurement at depth $p-1$,

$K=C_{X_{d}}(p-1) H^{t}\left[H C_{X_{d}}(p-1) H^{t}+R\right]^{-1}$,

$R$ is the covariance matrix of the detection error.

In order to obtain a fast algorithm, all the $v$ coordinates of the zones of interest are fixed and the detector used to extract a piece of road side or a road line is very simple. To obtain an optimal precision when depth $p f$ is reached, the algorithm looks for remaining zones of interest could not have been scanned yet. 


\subsection{Localization}

Once we have found the road in the image, we compute the new $X(k)$ vector from which we extract the $X_{l}$ vector. The method uses the result of road detection module $\hat{X}_{d}(k)$ and the old $X$ vector.

$\left\{\begin{array}{l}X(k)=X(k-1)+K\left[\widehat{X}_{d}(k)-H X(k-1)\right] \\ C_{X}(k)=C_{X}(k-1)-K H C_{X}(k-1)\end{array}\right.$

where

$\widehat{X}_{d}(k)=H X(k)$,
$K=C_{X}(k-1) H^{t}\left[H C_{X}(k-1) H^{t}+C_{X_{d}}(k-1)\right]^{-1}$.

\subsection{Tracking}

The goal of the tracking step is to obtain $X(k+1)$ and $C_{X}(k+1)$ from $X(k)$ and $C_{X}(k)$. This new vector and its covariance matrix will be used to initialize the first road detection step for the next image of the sequence. Tracking stage takes into account the displacement of the vehicle between two images (calculation of $\left.X_{l}(k+1)\right)$.

$$
\left\{\begin{array}{l}
X(k+1)=X(k)+K\left[X_{l}(k+1)-X_{l}(k)\right] \\
C_{X}(k+1)=C_{X}(k)-K H C_{X}(k)
\end{array}\right.
$$

where

$$
\begin{aligned}
& X_{l}=H X \\
& K=C_{X}(k) H^{t}\left[H C_{X}(k) H^{t}+C_{X_{l}}(k+1)\right]^{-1} .
\end{aligned}
$$

\section{ROAD MOTION ESTIMATION}

The previous step enabled us to detect area $R$ of the image corresponding to the road. Road motion $\vec{V}$ in image sequence $I(u, v, t)$ is performed by a wavelets analysis of the well known Brightness Change Constraint Equation :

$$
\begin{gathered}
\forall(u, v) \in R \\
\vec{\nabla} I((u, v), t) \cdot \vec{V}((u, v), t)+\frac{\partial I((u, v), t)}{\partial t}=0
\end{gathered}
$$

where $\vec{\nabla} I((u, v), t)$ and $\frac{\partial I}{\partial t}((u, v), t)$ are respectively gradient and temporal derivative.

In this article, we modelize the road motion by a 2D quadratic model with eight free parameters (Lefaix et al., 2002). This model can exactly represent the road motion due to the car motion. Let us note $\Theta=\left(a_{0}, a_{1}, a_{2}, a_{3}, a_{4}, a_{5}, a_{6}, a_{7}\right)^{T}$, the optical flow $\vec{V}_{\Theta}(u, v)$ at pixel $(u, v)$. The quadratic motion is given by :

$$
\begin{gathered}
\vec{V}_{\Theta}(u, v)=B(u, v) \Theta \quad \forall(u, v) \in R \\
B(u, v)=\left[\begin{array}{llllllll}
1 & 0 & u & v & 0 & 0 & u^{2} & u v \\
0 & 1 & 0 & 0 & u & v & u v & v^{2}
\end{array}\right]
\end{gathered}
$$

Let us consider the wavelets basis $\left(\Psi^{n}\right)_{i=1 \cdots N}$ in $L^{2}\left(\mathbf{R}^{2}\right)$ centered around the origin $(0,0)$, and let us consider the $\mathrm{N}$ functions centered around point $\left(2^{-j} k_{1}, 2^{-j} k_{2}\right)$ defined as :

$$
\Psi_{j k}^{n}(u, v)=2^{j} \Psi^{n}\left(2^{j} u-k_{1}, 2^{j} v-k_{2}\right),
$$

where $k=\left(k_{1}, k_{2}\right)$ and $j$ is the index of resolution. Taking the inner product of (5) with $\Psi_{j k}^{n}$, we obtain the following system :

$$
\left\langle\vec{\nabla} I \cdot \vec{V}+\frac{\partial I}{\partial t}, \Psi_{j k}^{n}\right\rangle=0 \quad \forall n=1 \cdots N,
$$

where

$$
\langle f, g\rangle=\iint f(u, v) \overline{g(u, v)} d u d v .
$$

That leads to :

$$
\begin{aligned}
& \forall n=1 . . N \\
& \left\langle\frac{\partial I}{\partial u} V_{1}, \Psi_{j k}^{n}\right\rangle+\left\langle\frac{\partial I}{\partial v} V_{2}, \Psi_{j k}^{n}\right\rangle+\left\langle\frac{\partial I}{\partial t}, \Psi_{j k}^{n}\right\rangle=0 .
\end{aligned}
$$

If we suppose $\vec{V}$ quadratic at this resolution $j$ with $\Theta^{j}=\left(a_{0}^{j}, a_{1}^{j}, a_{2}^{j}, a_{3}^{j}, a_{4}^{j}, a_{5}^{j}, a_{6}^{j}, a_{7}^{j}\right)^{T}$.

By substituting quadratic model (6) in system (8) and by integrating it by parts, we obtain the following system :

$$
\begin{aligned}
& a_{0}^{j}\left\langle I, \frac{\partial \Psi_{j k}^{n}}{\partial u}\right\rangle+a_{1}^{j}\left\langle I, \frac{\partial \Psi_{j k}^{n}}{\partial v}\right\rangle+a_{2}^{j}\left[\left\langle I, \Psi_{j k}^{n}\right\rangle+\left\langle u I, \frac{\partial \Psi_{j k}^{n}}{\partial u}\right\rangle\right]+ \\
& a_{3}^{j}\left\langle v I, \frac{\partial \Psi_{j k}^{n}}{\partial u}\right\rangle+a_{4}^{j}\left\langle u I, \frac{\partial \Psi_{j k}^{n}}{\partial v}\right\rangle+a_{5}^{j}\left[\left\langle I, \Psi_{j k}^{n}\right\rangle+\left\langle v I, \frac{\partial \Psi_{j k}^{n}}{\partial v}\right\rangle\right]+ \\
& a_{6}^{j}\left[\left\langle 3 u I, \Psi_{j k}^{n}\right\rangle+\left\langle u^{2} I, \frac{\partial \Psi_{j k}^{n}}{\partial u}\right\rangle+\left\langle u v I, \frac{\partial \Psi_{j k}^{n}}{\partial v}\right\rangle\right] \\
& +a_{7}^{j}\left[\left\langle 3 v I, \Psi_{j k}^{n}\right\rangle+\left\langle v^{2} I, \frac{\partial \Psi_{j k}^{n}}{\partial v}\right\rangle+\left\langle u v I, \frac{\partial \Psi_{j k}^{n}}{\partial u}\right\rangle\right]=\left\langle\frac{\partial I}{\partial t}, \Psi_{j k}^{n}\right\rangle \\
& \forall n=1 . . N, \forall(u, v)=\left(2^{-j} k_{1}, 2^{-j} k_{2}\right) \in R
\end{aligned}
$$

So, at fixed resolution $j$, we obtain $N$ equations for each point $\left(2^{-j} k_{1}, 2^{-j} k_{2}\right) \in R$. We note this system :

$$
M_{j} V_{\Theta^{j}}=P_{j}
$$

\subsection{Temporal aliasing}

As the image sequence is sampled in time, we have to estimate the temporal derivative of $I$ with a finite difference:

$$
\left\langle\frac{\partial I}{\partial t}, \Psi_{j k}^{n}\right\rangle \simeq\left\langle I(t+1)-I(t), \Psi_{j k}^{n}\right\rangle
$$

We can show (Bernard, 1998) that this approximation is valid if the optical flow verifies :

$$
\|\vec{v}\|<\text { Cst.size of wavelets support }
$$

So, at a fine scale, only the minor displacements can be estimated. Consequently, to compute the large displacements, we have to compute recursively an estimation of the flow at a coarse scale 
and estimate the residue between this value and the real flow in a finer scale.

Let us suppose that in a coarsest scale $j=J$, the solution of $(10)$ is $\Theta^{J}$. At scale $J+1$, we can decompose vector $\Theta$ as follows :

$$
\Theta=\mathcal{P}_{J \rightarrow J+1}\left(\Theta^{J}\right)+\Theta^{J+1}
$$

where $\mathcal{P}_{j \rightarrow j^{\prime}}$ is the projection of the scale $j$ on to the scale $j^{\prime}$ :

$$
\mathcal{P}_{j \rightarrow j^{\prime}}(\Theta)=\begin{aligned}
& \left(2^{j-j^{\prime}} a_{0}^{j}, 2^{j-j^{\prime}} a_{1}^{j}, a_{2}^{j}, a_{3}^{j}\right. \\
& \left., a_{4}^{j}, a_{5}^{j}, 2^{j^{\prime}-j} a_{6}^{j}, 2^{j^{\prime}-j} a_{7}^{j}\right)^{T}
\end{aligned}
$$

and where $\Theta^{J+1}$ is smaller than $\mathcal{P}\left(\Theta^{J}\right)$. Let us introduce $\widetilde{I}_{\Theta^{J}}(t+1)$ :

$$
\widetilde{I}((u, v), t+1)=I\left((u, v)+\vec{V}_{\mathcal{P}\left(\Theta^{J}\right)}, t+1\right) .
$$

The dominant motion between $I(., t)$ and $\widetilde{I}(., t+1)$ is exactly $V_{\Theta^{J+1}}$.

So, $\Theta^{J+1}$ is solution of system (10) where we replace $\left\langle\frac{\partial I}{\partial t}, \Psi_{j k}^{n}\right\rangle \simeq\left\langle I(t+1)-I(t), \Psi_{j k}^{n}\right\rangle$ by $\langle\widetilde{I}(t+$ 1) $\left.-I(t), \Psi_{j k}^{n}\right\rangle$. Then, we iteratively compute the optical flow by motion compensation from the coarsest scale at the finest scale.

Finally, we obtain an estimation of the dominant motion at each scale of the resolution :

$$
V_{\Theta^{j}}=B \cdot\left(\sum_{j^{\prime}=j}^{J} \mathcal{P}_{j^{\prime} \rightarrow j}\left(\Theta^{j^{\prime}}\right)+\Theta^{j}\right)
$$

\subsection{Robust estimation}

To solve system (10) at $N \times 2^{m+j} \times 2^{n+j}$ equations for eight unknowns and ensure robutness in the presence of independent motion, we use an Mestimator of Tukey, we look for $\widehat{V}_{\Theta^{j}}$ solution of :

$$
\begin{aligned}
& \widehat{V}_{\Theta^{j}}=\operatorname{argmin}_{V} \sum \rho\left(r_{i}, C\right) \\
& r_{i}=M_{j}(i) V(i)-Y_{j}(i)
\end{aligned}
$$

with,

$$
\rho(r, C)=\left\{\begin{array}{cl}
\frac{C^{2}}{6}\left(1-\left(1-\left(\frac{r}{C}\right)^{2}\right)^{3}\right) & \text { if }|r| \leq C \\
\frac{C^{2}}{6} & \text { else }
\end{array}\right.
$$

This minimization is performed using an iterative reweighted least squares procedure. This method allows us to get a robust estimation of the dominant motion at scale $j$.

\section{OBSTACLE DETECTION}

The previous step provides the road motion. As we have no a priori knowledge about the obstacle, we define an obstacle in the image as a set of pixels which are not in accordance with the road motion. To find these pixels, we can do a simple threshold. However, because of the noise in the image and the error of the optical flow estimation, this method is not sufficient. For this reason, we opt for a Bayesian template. But these methods are either too long in time (stochastic methods) or too dependent on initialization (determinist methods). Here, we choose a new Bayesian modelization : a hierarchical model (Pérez et al., 2000) which has the advantage to be fast and not very dependent on initialization.

Let us note $S=\cup_{n=0}^{J} S^{n}$ where $S^{i}$ indicates the level $i$ of the image resolution, $E=\left\{E_{s}, s \in S\right\}$ and $O=\left\{O_{s}, s \in S\right\}$ respectively the random field of the labels of the detected motion and the random field of the observations. $E_{s}$ can take two values, 0 or 1 , where 0 corresponds to a site non conform with the road motion and 1 to a site conform with this motion. Let us note $E^{n}$ the whole of the labels at level n, i.e. $E^{n}=\left\{E_{s}, s \in\right.$ $\left.S^{n}\right\}$ and in the same way $O^{n}=\left\{O_{s}, s \in S^{n}\right\}$. Finally, let us note $\bar{i}$ the parent of site $i, \underline{i}$ the whole of the children of $i$, and $\underline{i}$ the whole of the sites forming the tree of root $i$. Given this graphical structure, the distribution of $(E, O)$ can be written :

$$
\begin{aligned}
P(E= & e, O=o) \propto \exp -\left[\sum_{<i, j>\in S^{J}} v_{i, j}\left(e_{i}, e_{j}\right)\right. \\
& \left.+\sum_{i \notin S^{J}} w_{i}\left(e_{i}, e_{\bar{i}}\right)+\sum_{i \in S} l_{i}\left(e_{i}, o_{i}\right)\right]
\end{aligned}
$$

where $\langle i, j\rangle$ designates pairs of neighbors in $S^{J}, v_{i, j}$ and $w_{i}$ are local functions capturing respectively the spatial a priori and the hierarchical a priori and $l_{i}$ expresses the point-wise relation between observed variable $o_{i}$ and the unknown $e_{i}$. The associated MAP estimator at this distribution :

$$
\widehat{e} \in \operatorname{argmax}_{e} P(e \mid o)=\operatorname{argmax}_{e} P(e, o)
$$

is computed from the semi-iterative algorithm described in (Pérez et al., 2000). Let us note that the algorithm is applied only to one coarse resolution of the image where the data file is weaker. It is why this algorithm is less sensitive to initialization than a simple algorithm of deterministic minimization on the whole of the image.

Let us now interest to the field known on the image, called observations $Y$.

\subsection{Observations}

At a fixed scale $j$, we pose:

$$
E\left(j, k, \Theta^{j}\right)=\sum_{n=1}^{N}\left|\left\langle\vec{\nabla} I . \vec{V}_{\Theta^{j}}+\frac{\partial I}{\partial t}, \Psi_{j k}^{n}\right\rangle\right|^{2}
$$


We can show that : $\forall \epsilon>0$

$E\left(j, k, \Theta^{j}\right) \leq \lambda_{1} . \epsilon \Rightarrow\left\|V_{\Theta^{j}}-V_{j k}\right\| \leq \epsilon$

$E\left(j, k, \Theta^{j}\right) \geq \lambda_{2} . \epsilon \Rightarrow\left\|V_{\Theta^{j}}-V_{j k}\right\| \geq \epsilon$.

where $V_{j k}$ is the real flow at scale $j$ at the point $\left(2^{-j} k_{1}, 2^{-j} k_{2}\right)$ and $\lambda_{1}, \lambda_{2}$ respectively the smallest and the greatest eigenvalue of

$$
A=\left(\begin{array}{cc}
\sum_{n=1}^{N}\left|\left\langle I, \frac{\partial \Psi_{j k}^{n}}{\partial u}\right\rangle\right|^{2} & \sum_{n=1}^{N}\left\langle I, \frac{\partial \Psi_{j k}^{n}}{\partial u}\right\rangle\left\langle I, \frac{\partial \Psi_{j k}^{n}}{\partial v}\right\rangle \\
\sum_{n=1}^{N}\left\langle I, \frac{\partial \Psi_{j k}^{n}}{\partial u}\right\rangle\left\langle\overline{\left\langle, \frac{\partial \Psi_{j k}^{n}}{\partial v}\right\rangle}\right. & \sum_{n=1}^{N}\left|\left\langle I, \frac{\partial \Psi_{j k}^{n}}{\partial v}\right\rangle\right|^{2}
\end{array}\right)
$$

We will note $\lambda_{1} . \epsilon\left(\right.$ resp. $\left.\lambda_{2} . \epsilon\right), l_{j k}$ (resp. $\left.L_{j k}\right)$. Consequently, $E\left(j, k, \Theta^{j}\right)$ translates the error made by approaching the real optical flow at point $\left(2^{-j} k_{1}, 2^{-j} k_{2}\right)$ by $V_{\Theta^{j}}$. If $E\left(j, k, \Theta^{j}\right) \leq l_{j k}$, we can affirm that the error is smaller than $\epsilon$ and if $E\left(j, k, \Theta^{j}\right) \geq L_{j k}$, the error is larger than $\epsilon$. This error will enable us to judge if point $\left(2^{-j} k_{1}, 2^{-j} k_{2}\right)$ follows the dominant motion.

\subsection{The choice of potentials}

For the space and hierarchical potentials, we choose a priori classical functions of Potts :

$$
\begin{gathered}
v_{i, j}\left(e_{i}, e_{j}\right)=\alpha\left[1-\delta\left(e_{i}, e_{j}\right)\right], \\
w_{i}\left(e_{i}, e_{\bar{i}}\right)=\beta\left[1-\delta\left(e_{i}, e_{\bar{j}}\right)\right] .
\end{gathered}
$$

These potentials favour the labels field of detection being homogeneous in space and in scale. Energy $l_{i}$ is defined by :

$$
\begin{aligned}
& \forall i=\left(2^{-j} k_{1}, 2^{-j} k_{2}\right) \in S^{j} \\
& l_{i}\left(e_{i}, e_{i}\right)= \\
& \begin{cases}\frac{1}{1+\exp \left(E\left(j, k, \Theta^{j}\right)-l_{j k}\right)} & \text { if } e_{i}=0 \\
1-\frac{1}{1+\exp \left(E\left(j, k, \Theta^{j}\right)-L_{j k}\right)} & \text { if } e_{i}=1\end{cases}
\end{aligned}
$$

This potential encourages the sites to take label 1 (conform to motion dominant) in the case where $E\left(j, k, \Theta_{D}^{j}\right) \leq l_{j k}$ and to take label 0 (non conform to motion dominant) if $E\left(j, k, \Theta_{D}^{j}\right) \geq L_{j k}$.

\section{RESULTS}

For our experimentations, we use $360 \times 280$ (highways images) and $256 \times 256$ (other road images) pixel image size. The used camera has a focal length of $f=11.48 \mathrm{~mm}$ and its position is on the top of the vehicle $\left(Z_{0}=2.32 m\right)$. This high position authorizes a 3D shape road reconstruction of one hundred meters in front of the vehicle.

For the training step, because we have two types of images (highways sequences(h.) and other

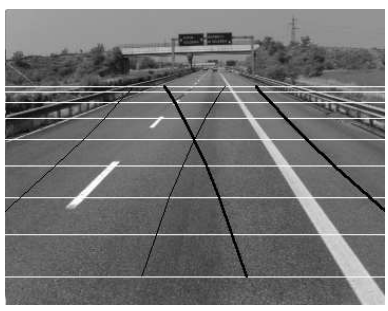

(a)

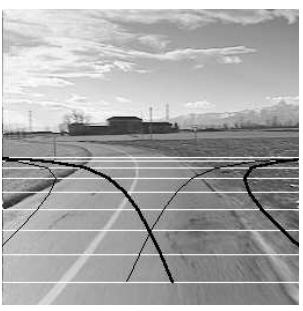

(b)
Fig. 2. Initial search zone obtained after the training step for highways (a) and semi-marked roads $(b)$.

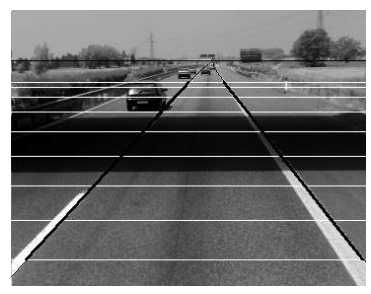

(a)

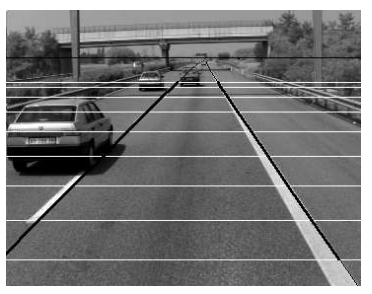

(b)
Fig. 3. Detection road result for highways and 3D projected model with different conditions.

roads sequences (r.)), we use two sets of data :

\begin{tabular}{|c|c|c|c|c|c|}
\hline & $\alpha$ (deg.) & $\psi$ (deg.) & $C\left(m^{-1}\right)$ & $L(m)$ & $x_{0}(m)$ \\
\hline$\mu(h)$. & 4.5 & 0 & 0 & 4 & 2 \\
\hline$\mu(r)$. & 0.9 & 0 & 0 & 3.5 & 1.75 \\
\hline$\sigma(h)$. & 0.5 & 3 & 0.0001 & 0.5 & 2 \\
\hline$\sigma(r)$. & 0.5 & 3 & 0.005 & 0.5 & 1.75 \\
\hline
\end{tabular}

In Figure 2, training results are shown. Horizontal limits of the search zone are a priori fixed and represented as white lines. We can see the eight trapezoidal zones of interest found for the left road side/line (fine black line for both vertical limits) and the right road side/line (large black line).

In Figures 3 and 4, we can see both recognition results (large black line) of the road detection module and a 3D projected model (fine black line) of the road after the localization step. For all types of road, in most situations, the number of valid detected segments is never lower than 11 in spite of obstacles or road occlusions. Concerning the projected model, the errors made in the estimation of curvature parameter $C$ and vertical angle camera $\alpha$ at localization stage introduce an error on the road localisation in the upper part of the image. Nevertheless, this approximation is sufficient for our application. For the parameters of the hierarchical model, we fix $\alpha=8, \beta=100$. In the highway sequence, error $\epsilon=2$ and in road sequence $\epsilon=1.2$.

In Figures 5 and 6 , we can see the results of the obstacle detection where the rectangles include the dense areas detected as obstacles. Generally we detect the obstacles correctly. In the highway sequence (Fig 5), the vehicle in the foreground, and in the road sequence (Fig 6), the truck are correctly detected. But for example, in the image $t=31$ in the highway sequence, we do not de- 


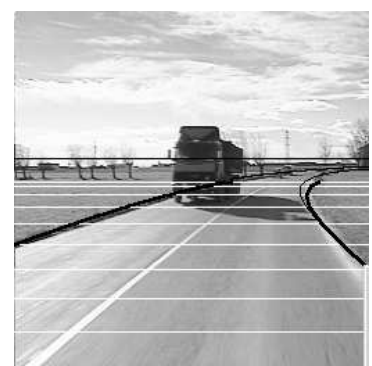

(a)

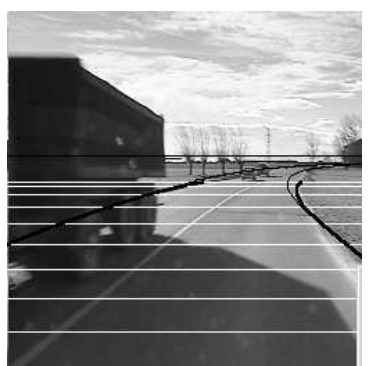

(b)
Fig. 4. Detection road result for semi-marked roads and 3D projected model.

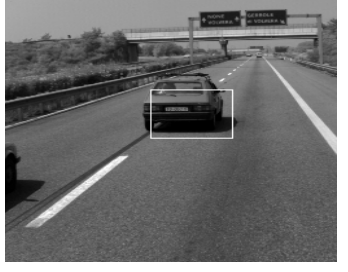

(a)

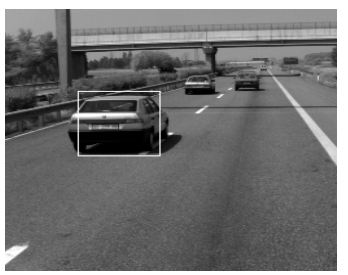

(c)

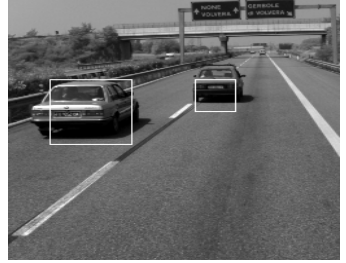

(b)

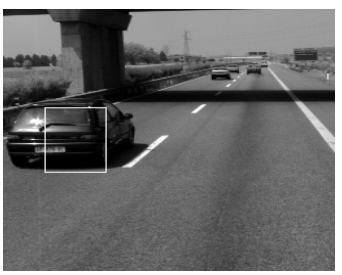

(d)
Fig. 5. Obstacle detection on the highway sequence. $\alpha=8, \beta=100, \epsilon=2$. (a) $t=10$, (b) $t=15$, (c) $t=31$, (d) $t=59$.

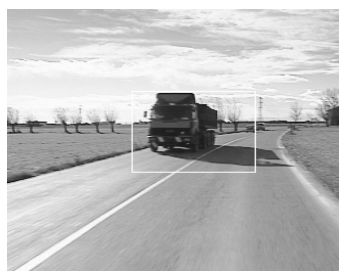

(a)

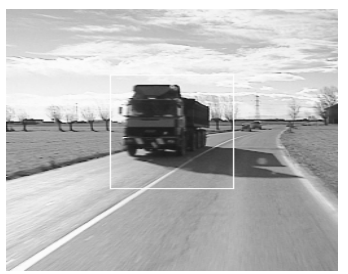

(b)
Fig. 6. Obstacle detection on the road sequence. $\alpha=8, \beta=100, \epsilon=1.2$. (a) $t=66$, (b) $t=70$.

tect the vehicles in the background. It's because these vehicles are too far and at this distance the difference between their real velocity in the image and the road motion is lower than $\epsilon$. To solve this problem, we will incorporate in a future work a tracking of the labels field in time.

\section{CONCLUSION AND FUTURE WORKS}

In this work, a vision technique for obstacle detection has been proposed. The originality of this work lies in the exploitation of motion to obtain a robust search and tracking of moving obstacles. Initially, the road is detected in the sequence through a recursive algorithm with a probabilistic road model. A second phase based on motion analysis is performed using wavelets approach and a Bayesian hierarchical model. This vision system is fast and robust. Indeed, the tests have proved that it is possible to detect any obstacle on the road in presence of shadows, occlusions or varying illumination conditions. However, in future works it will be interesting to add a tracking of the labels field in time in order to limit the number of false alarms and especially to detect obstacles in the background.

\section{REFERENCES}

Bernard, C. (1998). Discrete wavelet analysis: A new framework for fast optic flow computation. In: $E C C V 98$.

Bertozzi, M. and A. Broggi (1998). Gold: A parallel real-time stereo vision system for generic obstacle and lane detection. IP 7(1), 62-81.

Broggi, A., M. Bertozzi, A. Fascioli and M. Sechi (2000). Shape-based pedestrian detection. In: IEEE Intelligent Vehicles Symposium'00. pp. 215-220.

Chapuis, R., R. Aufrère and F. Chausse (2002). Accurate road following and reconstruction by computer vision. Transactions on Intelligent Transportation Systems 3(4), 261-270.

Chausse, F., R. Aufrère and R. Chapuis (2000). Recovering the $3 \mathrm{~d}$ shape of a road by onboard monocular vision. In: ICPRO0. pp. Vol I: $325-328$.

Kruger, W., W. Enkelmann and S. Rossle (1995). Real-time estimation and tracking of optical flow vectors for obstacle detection. In: IEEE Intelligent Vehicles Symposium'95. pp. 304309.

Kuehnle, A. (1998). Symmetry-based vehicle location for ahs. In: Procs. SPIE - Transportation Sensors and Controls : Collision Avoidance, Traffic Management, and ITS. Vol. 2902. pp. 19-27.

Lefaix, G., E. Marchand and P. Bouthemy (2002). Motion-based obstacle detection and tracking for car driving assistance. In: ICPR02. Vol. 4. pp. 74-77.

Lutzeler, M. and E.D. Dickmanns (1998). Road recognition with marveye. In: IEEE Intelligent Vehicles Symposium'98. pp. 341-346.

Pérez, P., A. Chardin and J.M. Laferte (2000). Noniterative manipulation of discrete energybased models for image analysis. $P R$ 33(4), 573-586. 